\title{
A Case Report of a Very Rare Association of Tyrosinemia type I and Pancreatitis Mimicking Neurologic Crisis of Tyrosinemia Type I
}

\author{
Habibe Koç Uçar ${ }^{1}$, Gökhan Tümgör ${ }^{1}$, Deniz Kör ${ }^{1}$, Fatih Kardaş ${ }^{2}$, Neslihan Önenli Mungan ${ }^{1}$ \\ ${ }^{1}$ Department of Pediatrics, Çukurova University School of Medicine, Adana, Turkey \\ ${ }^{2}$ Department of Pediatrics, Erciyes University School of Medicine, Kayseri, Turkey
}

Background: Tyrosinemia type I is an autosomal recessively inherited metabolic disease of tyrosine metabolism due to the deficiency of fumarylacetoacetate hydrolase. Clinical manifestations include hepatic failure, cirrhosis, hepatocellular carcinoma, renal fanconi syndrome, and neurologic crisis. With the introduction of 2-(2-nitro-4-trifluoro-methylbenzyol)-1.3 cyclohexanedione (NTBC) treatment, the prognosis improved with reduced rate of complications.

Case Report: Here, we report a 6-year-old girl with tyrosinemia type I who discontinued NTBC treatment six months prior to admission, presenting with com- plaints of abdominal pain, vomiting, anorexia, weakness, and restlessness, suggesting the clinical status of neurologic crisis. Further laboratory and radiologic evaluation revealed that indeed this is a pancreatitis.

Conclusion: We report this case as tyrosinemia type I and pancreatitis was reported only in one case in the literature, emphasizing confusing clinical signs of neurological crisis, and pancreatitis in tyrosinemia type I.

Keywords: Neurologic crisis, pancreatitis, tyrosinemia type I
Tyrosinemia type I is a metabolic disease of tyrosine metabolism due to the deficiency of fumarylacetoacetate hydrolase (FAH) enzyme which is inherited in an autosomal recessive manner and is characterized by severe hepatocellular degeneration (1). This disorder was first described by Sakai and Kitogawa in 1957 (2). The incidence is about 1/100000 (3). Clinical manifestations of untreated cases are hepatic failure, cirrhosis, hepatocellular carcinoma, renal fanconi syndrome, and neurologic crisis. After introducing Nitisinone (2-(2-nitro-4-trifluoro-methylbenzyol)-1.3 cyclohexanedione) (NTBC) (Orfadin; Sobi, Stockholm, Sweden) to the treatment and with the restriction of tyrosine and phenylalanine in the diet, the prognosis improves and a limited number of complications have been reported. This treatment can prevent neurologic crises, acute hepatic failure, and renal tubular acidosis (4). Neurologic crisis is a rare but fatal complication of tyrosinemia type I characterized by anorexia, vomiting, and sometimes self-mutilation, with hyponatremia in the initial phase, and continuing with paresthesia, autonomic symptoms and possible progression to paralysis of the extremities and diaphragm and death.

Acute pancreatitis is transient inflammation of the pancreas. The diagnosis is made with two of the three criteria listed below: clinical symptoms of severe abdominal pain, vomiting, elevation of serum and urine amylase or lipase levels three times from the upper limit and radiological evidence of pancreatitis by ultrasonography or tomography. The etiology of pancreatitis in children is quite diverse: anatomical anomalies, drugs, trauma, biliary problems, genetic causes, hyperlipidemia, organic acidemia, and postsurgical problems of the gastrointestinal system. When it is possible to rule out all causes, an idiopathic pancreatitis diagnosis can be made (5). In the literature, tyrosinemia type I with pancreatitis was only reported in one case (6). 
TABLE 1. Course of laboratory findings during hospitalization

\begin{tabular}{|c|c|c|c|c|}
\hline \multirow[b]{2}{*}{ Laboratory Values } & \multicolumn{3}{|c|}{ Date } & \multirow[b]{2}{*}{$\begin{array}{l}\text { On the } 13^{\text {th }} \text { day of } \\
\text { hospitalization }\end{array}$} \\
\hline & $\begin{array}{l}\text { On the } 1^{\text {st }} \text { day of } \\
\text { hospitalization }\end{array}$ & $\begin{array}{l}\text { On the } 7^{\text {th }} \text { day of } \\
\text { hospitalization }\end{array}$ & $\begin{array}{l}\text { On the } 9^{\text {th }} \text { day of } \\
\text { hospitalization }\end{array}$ & \\
\hline $\mathrm{Na}(\mathrm{mmol} / \mathrm{L}) \mathrm{N}: 135-145$ & 127 & & 136 & 136 \\
\hline $\mathrm{K}(\mathrm{mmol} / \mathrm{L}) \mathrm{N}: 3.5-5.1$ & 2.9 & & 5 & 4.9 \\
\hline Glucose (mg/dL) N: 70-105 & 98 & & 69 & 90 \\
\hline $\operatorname{AST} / \operatorname{ALT}(\mathrm{U} / \mathrm{L}) \mathrm{N}: 0-31 / 0-31$ & $32 / 21$ & & & $25 / 22$ \\
\hline $\operatorname{ALP}(\mathrm{U} / \mathrm{L}) \mathrm{N}: 38-126$ & 91 & & & 124 \\
\hline $\operatorname{AFP}(\mathrm{ng} / \mathrm{mL}) \mathrm{N}: 0-6$ & 42.07 & & & 6 \\
\hline Serum Lipase (U/L) N: 22-51 & & 185 & 95 & 20 \\
\hline Serum Amylase (U/L) N: 28-100 & & 312 & 271 & 85 \\
\hline Urine Amylase (U/L) N: 21-447 & & 731 & 637 & 64 \\
\hline
\end{tabular}

Na: sodium; K: potassium; AST: aspartate transferase; ALT: alanine aminotransferase; ALP: alkaline phosphatase; AFP: alpha fetoprotein

Here, we report a case of tyrosinemia type I and pancreatitis, as pancreatitis could be a rare underestimated complication of tyrosinemia type I and our patient has a confusing clinical manifestation, mimicking neurologic crisis.

\section{CASE PRESENTATION}

A 6-year-old girl was admitted to the emergency room with complaints of severe abdominal pain, vomiting, anorexia, weakness, and restlessness which began two weeks earlier and had progressed. She was diagnosed with tyrosinemia type I due to a homozygous IVS6-1G>T mutation in the FAH gene at the age of two years, and she was put on NTBC (Orfadin; Sobi, Stockholm, Sweden) treatment. Six months before admission, the family self-discontinued NTBC (Orfadin; Sobi, Stockholm, Sweden).

The child was born at term as a first child of a non-consanguineous parents with a normal birth weight and length. She maintained normal physical, mental, and motor growth on NTBC (Orfadin; Sobi, Stockholm, Sweden) treatment and never required hospitalization after the first attack. On physical examination she presented a weight of $75-90 \%$, height of $25 \%$, normal blood pressure, slight fever, tachypnea and tachycardia. She appeared anxious, and ill and was in lateral knee-chest position due to the general tenderness in the abdomen; however, defense, rebound, organomegaly and ascites were not present. Laboratory examination revealed normal hepatic and renal function tests and acute phase reactants. There were no clinical or laboratory signs of viral infection. There was no history of trauma. Hyponatremia and natriuresis were determined. Blood tyrosine and alpha-fetoprotein levels were slightly elevated. Abdominal ultrasonography was normal (Table 1).
In the follow-up, axillary fever was $38.3^{\circ} \mathrm{C}$ with no signs of infection; this was considered an indicator of autonomic dysfunction. On the second day, episodes of generalized tonicclonic seizures were observed. There was a slowing of background rhythm on electroencephalography. With the history of NTBC (Orfadin; Sobi, Stockholm, Sweden) discontinuation and clinical and laboratory findings, a neurologic crisis was initially diagnosed, and NTBC (Orfadin; Sobi, Stockholm, Sweden) treatment was restarted at a dose of $1 \mathrm{mg} / \mathrm{kg} /$ day with a tyrosine and phenylalanine restricted diet. As her electromyography (EMG) was normal, and abdominal pain persisted, other probable causes of abdominal pain were considered. High levels of urine and serum amylase and lipase and homogeneous enlargement and edema of the pancreatic head on abdominal tomography indicated acute pancreatitis. As other probable causes of pancreatitis had been excluded, a very rare case of acute pancreatitis due to tyrosinemia type I was diagnosed. Clinical status, serum and urine amylase and lipase levels returned to normal levels on the $13^{\text {th }}$ day of treatment. The patient was discharged with the importance of maintaining NTBC therapy (Orfadin; Sobi, Stockholm, Sweden) being reemphasized. An informed consent was taken from the patient's parent.

\section{DISCUSSION}

Neurologic crisis was frequently seen in tyrosinemia type I before NTBC (Orfadin; Sobi, Stockholm, Sweden) treatment. In 1990, Lindstedt et al. (7) reported that neurological crisis developed in $42 \%$ of 48 children with tyrosinemia type I during the first year of life. In 2008, Schlump et al. (8) reported that all neurological signs and symptoms improved after restarting 
NTBC (Orfadin; Sobi, Stockholm, Sweden) treatment in an 8 month-old male who had a severe neurological crisis with progressive ascendant polyneuropathy, diaphragm paralysis, arterial hypertension, and respiratory distress after the discontinuation of NTBC (Orfadin; Sobi, Stockholm, Sweden) treatment (8). Neurologic crisis in tyrosinemia type I is similar to attacks of acute intermittent porphyria, which begins with anorexia, vomiting, and abdominal pain and isfollowed by painful paresthesias and paralyses, typically after a mild infection. Death was inevitable in cases without treatment. Neurological crisis was reported in a limited number of cases after the introduction of NTBC (Orfadin; Sobi, Stockholm, Sweden) into the current management (6). Discontinuation of NTBC (Orfadin; Sobi, Stockholm, Sweden) treatment followed by abdominal pain, vomiting, weakness and irritability suggested a neurologic crisis in our case. Hyponatremia and natriuresis supported the diagnosis. However, normal levels of urinary 5-deltaaminolevulinic acid and succinylacetone together with normal EMG findings ruled out neurologic crisis. As we continued to investigate other causes of abdominal pain, elevated levels of amylase and lipase in urine and serum indicated a clinical picture of acute pancreatitis. We could not find any etiological factor that can cause acute pancreatitis other than tyrosinemia type I. In the literature, Kalkanoglu et al. (6) reported the only case of an acute pancreatitis with tyrosinemia type I. Like them, we could not offer an explanation for the occurrence of these two entities. Our patient's clinic dramatically improved with NTBC (Orfadin; Sobi, Stockholm, Sweden) and supportive treatments and did not recur in the following months.

Here, we report this case, where pancreatitis in tyrosinemia type I presents a confusing clinical picture and was reported only once. Additionally, this case shows that acute pancreatitis should be taken into consideration in the differential diagnosis in cases with tyrosinemia presenting with features like porphyria or neurologic crisis. Further studies are necessary to explain the pathophysiology of the development of pancreatitis in tyrosinemia type I.

\section{Ethics Committee Approval: N/A.}

Informed Consent: Written informed consent was obtained from the parent of the patient who participated in this case.

Peer-review: Externally peer-reviewed.
Author contributions: Concept - G.T., D.K., N.Ö.M.; Design G.T., D.K., N.Ö.M.; Supervision - G.T., D.K., N.Ö.M.; Resource H.K.U., D.K.; Materials - H.K.U., G.T., D.K., F.K., N.Ö.M.; Data Collection and/or Processing - H.K.U., D.K., F.K.; Analysis and/or Interpretation - D.K., F.K.; Literature Search - H.K.U., D.K.; Writing - H.K.U., D.K.; Critical Reviews - H.K.U., G.T., D.K., F.K., N.Ö.M.

Conflict of Interest: No conflict of interest was declared by the authors.

Financial Disclosure: The authors declared that this study has received no financial support.

\section{REFERENCES}

1. Nyhan WL, Barshop BA, Al-Aqeel AI. Hepatorenal tyrosinemia/ fumarylacetoacetate hydrolase deficiency. In: Nyhan WL, Barshop BA, Al-Aqeel AI. Atlas of inherited metabolic diseases. 3rd ed. London: Hodder Arnold and Hachette UK Company. 2012:171-9.

2. Chakrapani A, Gissen P, McKiernan P. Disorders of tyrosine metabolism. In: Saudubray JM, Berghe GVD, Walter JH. Inborn Metabolic Disease. 5th ed. Germany; Springer. 2012:265-77. [CrossRef]

3. Rezvani I, Mitchel G. Tyrosine. In: Kliegman RM, Behrman RE, Jenson HB, Stanton BMD. Nelson Textbook of Pediatrics. 18th ed. Philadelphia: Saunders Elsevier. 2007;532.

4. Santra S, Preece MA, SA Hulton, McKiernan PJ. Renal tubular function in children with tyrosinaemia type I treated with nitisinone. J Inherit Metab Dis 2008;31:399-402. [CrossRef]

5. Lowe ME. Pancreatitis. In: Wyllie R, Hyams JS, Kay M. Pediatric Gastrointestinal and Liver Disease. 4th ed. Philadelphia; Elsevier Saunders 2011;905-14.

6. Kalkanoğlu HS, Coşkun T. Neurological crisis mimicking acute pancreatitis in tyrosinemia type I. Turk J Pediatr 1999;41:501-4.

7. Lindstedt S, Holme E, Lock EA, Hjalmarson O, Strandvik B. Treatment of hereditary tyrosinaemia type I by inhibition of 4-hydroxyphenylpyruvate dioxygenase. Lancet 1992;340:8137. [CrossRef]

8. Schlump JU, Perot C, Ketteler K, Schiff M, Mayatepek E, Wendel $U$ et al. Severe neurological crisis in a patient with hereditary tyrosinaemia type I after interruption of NTBC treatment. J Inherit Metab Dis 2008;31:223-5. [CrossRef] 